\title{
Long Noncoding RNA MEG3 Inhibits Apoptosis of Retinal Pigment Epithelium Cells Induced by High Glucose via the miR-93/Nrf2 Axis
}

Rong Luo, Han Jin, Lan Li, Yu-Xiang Hu, and Fan Xiao

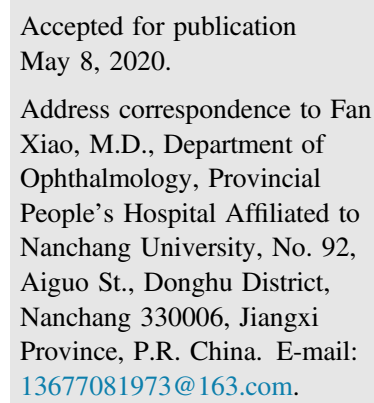

\begin{abstract}
Diabetic retinopathy (DR) is the leading cause of visual impairment in developed nations. Though plasma microRNA-93 (miR-93) is associated with the risk of DR, the function and regulatory mechanism of miR-93 during DR remains unclear. Blood samples were collected from 12 DR patients and 12 healthy controls. Primary human retinal pigment epithelium (RPE) cells and ARPE-19 cells were cultured in 5 $\mathrm{mmol} / \mathrm{L}$ or $33 \mathrm{mmol} / \mathrm{L}$ D-glucose medium. Long noncoding (lnc) RNA MEG3 and miR-93 expression was detected by real-time quantitative PCR. The effect of MEG3 and miR-93 on high glucose (HG)-induced apoptosis was detected by MTT and flow cytometry. IL- 6 and tumor necrosis factor- $\alpha$ levels were detected by enzyme-linked immunosorbent assay. The relationships among MEG3, miR-93, and Nrf2 (also known as NFE2L2) were explored via dual-luciferase reporter assay. IncRNA MEG3 and Nrf2 were decreased and miR-93 was increased in blood samples of DR patients and HG-treated human RPE and ARPE-19 cells. Overexpression of miR-93 inhibited cell proliferation and promoted apoptosis, whereas overexpression of Nrf2 or MEG3 promoted proliferation and suppressed apoptosis and inflammation. In addition, MEG3 targeted miR-93 and down-regulated miR-93. Moreover, miR-93 directly targeted Nrf2 and negatively regulated Nrf2. This study suggests that IncRNA MEG3 depresses HG-induced apoptosis and inflammation of RPE via miR-93/Nrf2 axis, providing a novel perspective on the genesis and development of DR. (Am J Pathol 2020, 190: 1813-1822; https://doi.org/10.1016/ j.ajpath.2020.05.008)
\end{abstract}

As the leading cause of blindness in Europe, diabetic retinopathy (DR) affects nearly $33.3 \%$ of diabetes mellitus patients ${ }^{1}$ and is the major cause of visual impairment in populations between 20 and 70 years of age. ${ }^{2}$ The current therapy for DR mainly consists of medication [intravitreous injection of glucocorticoids or vascular endothelial growth factor (VEGF) antagonists], laser photo coagulation, as well as early surgical treatment. ${ }^{3}$ Nowadays, the standard of care treatment for sight-threatening retinopathy remains retinal laser photocoagulation, though this treatment often cannot fully restore patients' lost vision., ${ }^{2,4}$

Retinal pigmented epithelium (RPE) is a special epithelium located at the junction of neural retina and choriocapillaris, where the external blood retinal barrier is formed. ${ }^{5}$ The tight junctions in both the endothelial and RPE barriers are compromised in DR. ${ }^{6-8}$ Diabetes and hypoxia (occurring in late stage of DR) induces the breakdown of the RPE barrier. ${ }^{8,9}$ Ultrastructural changes in the RPE can be readily detected during the early stage of diabetes. ${ }^{10-12}$ As a result of a diabetes-induced relaxation of the RPE barrier, an increased leakage of blood content occurrs. ${ }^{6,13}$ In addition, hyperglycemia or high glucose (HG)-induced apoptosis in human RPE cells is a characteristic process in DR. ${ }^{14}$ Therefore, the RPE is an important tissue biochemically and mechanistically in DR. In vitro studies in the last few years have shown that RPE cells play a significant part during the immune response through the expression of FasL, adhesion molecules, major histocompatibility complex molecules, and cytokines. ${ }^{15}$ Therefore, the role of RPE in regulating retinal immune response is indispensable under

Disclosures: None declared. 
normal physiological conditions, and is becoming more and more important in degenerative retinal diseases in which chronic subclinical inflammation is considered to be the main pathogenic factor. ${ }^{16,17}$ Early detection and treatment of the disease can decrease the risk of developing complications. Therefore, studying the mechanism of apoptosis and regulation of RPE cells will provide theoretical basis for potential therapeutic targets of DR.

As a group of RNA transcripts, long noncoding RNA (lncRNAs) are not translated into protein products. ${ }^{18}$ Studies over the past few years have also pointed out that lncRNA is also involved in the development of DR. ${ }^{19}$ Furthermore, a significantly lower level of maternally expressed gene 3 (MEG3) was observed in diabetic mice and patients than healthy controls. ${ }^{20}$ However, there are still many unknowns regarding the function and molecular mechanism of MEG3 during the progression of DR.

It is well known that the miRNAs are involved in the pathogenesis of diabetes and relevant micro- and macrovascular complications, and play an important role in many pathophysiological processes, for example, glucose angiogenesis, homeostasis, and the regulation of the inflammatory response. ${ }^{21}$ However, little has been reported about direct involvement of miRNA during DR. ${ }^{21}$ Lin et $\mathrm{al}^{14}$ have confirmed that miR-29 plays a vital role in the HG-induced apoptosis of RPE cells through the inverse correlation of PTEN. Twenty-four hours after the onset of diabetes, a down-regulated level of miR-126 was noticed in diabetic retinas of streptozotocin-induced diabetic rats. ${ }^{22}$ A previous study illustrated that miR-195 is up-regulated and SIRT1 is down-regulated in human retinal microvascular endothelial cells exposed to $\mathrm{HG}^{23}$ A significantly higher level of miR-93 (MIR93) was noticed in eyes with proliferative DR in another study, suggesting that miR-93 may have a role in angiogenesis and fibrosis. ${ }^{24}$

In view of miR-93 as an important regulator of DR, this study aimed to further examine the latent function of miR-93 during the pathological process of DR.

\section{Materials and Methods}

\section{Patients}

Twelve DR patients with a mean course of disease for $8.23 \pm 2.14$ years were included in this study. All of the patients were diagnosed according to the diagnostic criteria established by the Chinese Medical Association in 2014. Inclusion criteria: after routine fundus examination and fundus fluorescence angiography examination, type 2 diabetes mellitus patients with any one of the following conditions: hemangioma, a few small bleeding points, neovascularization, vitreous hemorrhage, or secondary retinal detachment in the retina. Exclusion criteria: patients with acute complications such as diabetic ketosis, hyperglycemic coma, severe stress such as recent cardiovascular events, trauma operation, acute or chronic infection, hepatic disease, and other endocrine metabolic disease. Meanwhile, 12 healthy controls were selected as a control group. No significant differences were found between the two groups in terms of age and sex. This study was approved by the Ethics Committee of Provincial People's Hospital Affiliated to Nanchang University. All patients signed informed consent. Fasting blood $(20 \mathrm{~mL})$ was extracted from each participant in the morning. Blood samples were kept at room temperature for 1 hour, followed by centrifugation at $2500 \times g$ for 20 minutes to collect plasma samples.

\section{Cell Culture}

Primary human RPE (HRPE) cells were purchased from Cell Systems (Kirkland, WA) and cultured in an endothelial growth medium-2 kit (Lonza, Basel, Switzerland). The human RPE cell line ARPE-19 was obtained from the Type Culture Collection of the Chinese Academy of Sciences (Shanghai, China). The complete culture medium for ARPE-19 cells consisted of Dulbecco's modified Eagle's medium/F-12 (volumetric ratio of 1:1; Hyclone, Logan, UT) and $10 \%$ fetal bovine serum (Hyclone). ARPE-19 and HRPE cells were cultured at $37^{\circ} \mathrm{C}$ in humidified air with $5 \%$ $\mathrm{CO}_{2}$. The culture medium was renewed every other day. For HG treatment, ARPE-19 and HRPE cells were grown in complete culture medium until approximately $75 \%$ confluent. Subsequently, the culture medium was replaced by fetal bovine serum-free Dulbecco's modified Eagle's medium/F-12 for 24 hours before switching to the HG condition ( $33 \mathrm{mmol} / \mathrm{L}$ D-glucose). The cell culture medium was not renewed during the 48 hours of HG treatment. When the cells were routinely cultured, the medium was renewed every other day.

\section{Quantitative Real-Time PCR Analysis}

Total RNA was extracted according to the manufacturer's instructions. Then, $1 \mu \mathrm{g}$ of RNA was reverse transcribed to cDNA with the Prime Script RT Kit (Fermentas, Burlington, ON, Canada). Quantitative real-time PCR (qPCR) was performed by using the SYBR Premix Ex Taq (Takara Bio Inc., Kusatsu, Japan) with a Roche 480 Light Cycler (Roche, Mannheim, Germany). The threshold cycle for each reaction was recorded as quantity of gene expression. The levels of target mRNA expression were analyzed by the comparative $2^{-\triangle \triangle \mathrm{Ct}}$ method using glyceraldehyde3-phosphate dehydrogenase $(G A P D H)$ as a control, whereas the miR-93 expression was calculated using U6 as a control.

\section{Western Blot Analysis}

After the indicated treatment, cells were lysed with radioimmunoprecipitation assay buffer, and then centrifuged. The protein concentrations were measured by using a BCA kit (Pierce, Rockford, Illinois). The protein sample was separated by $8 \%$ SDS-PAGE gels, and then they were 
A

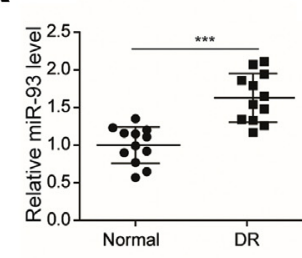

D
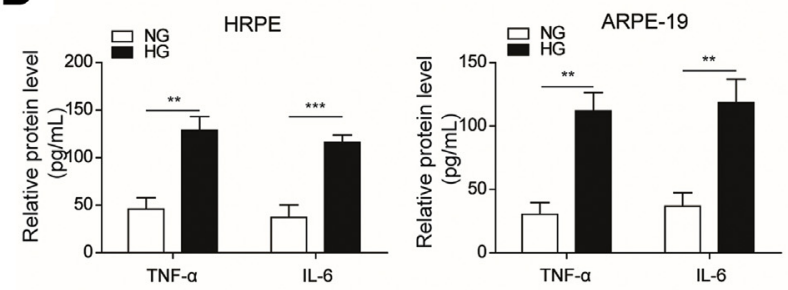

E

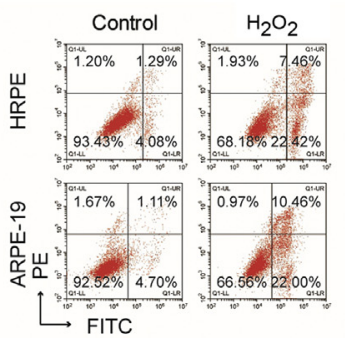

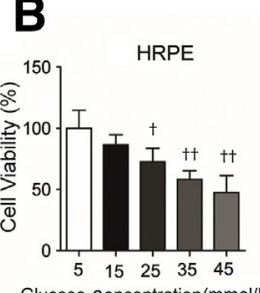

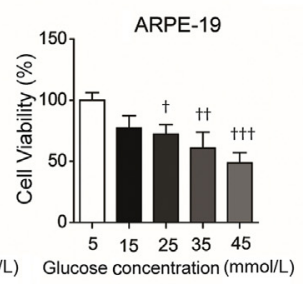

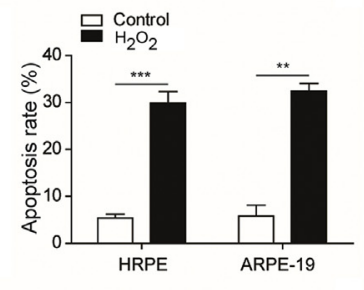
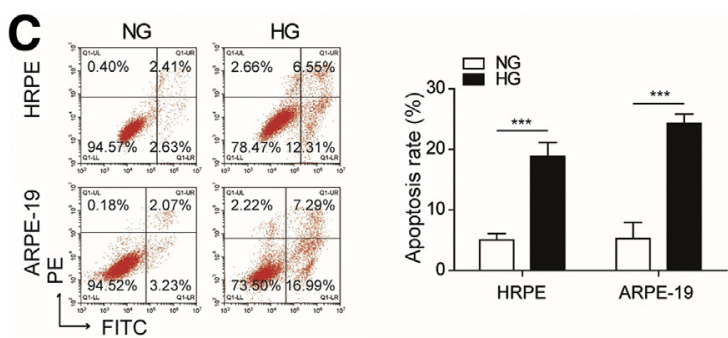

$\mathbf{E}$
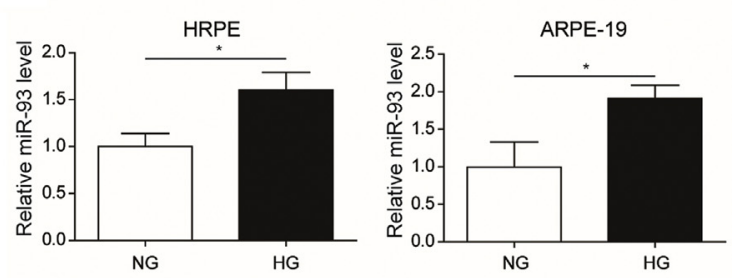

G

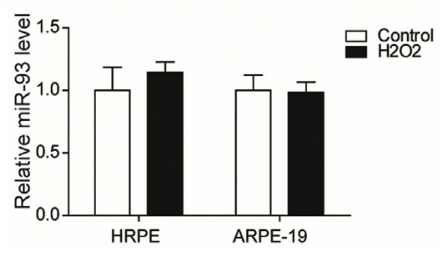

Figure 1 High glucose (HG) damages retinal pigment epithelium (RPE) cells and up-regulates miR-93 expression. A: Relative expression of miR-93 (MIR93) in plasma samples of normal controls and diabetic retinopathy (DR) patients. B: Cell viability of human primary RPE cells (HRPE) and ARPE-19 cells cultured in different concentrations of D-glucose $(5,15,25,35$, and $45 \mathrm{mmol} / \mathrm{L})$ for 48 hours. C: Apoptosis rate of HRPE and ARPE-19 cells cultured in $5 \mathrm{mmol} / \mathrm{L}$ (NG group) and $33 \mathrm{mmol} / \mathrm{L}$ (HG group) D-glucose for 48 hours. D: Expression of inflammatory factors IL- 6 and tumor necrosis factor (TNF)- $\alpha$ in NG and HG groups detected by enzyme-linked immunosorbent assay (ELISA). E: Relative expression of miR-93 in HRPE and ARPE-19 cells cultured in $5 \mathrm{mmol} / \mathrm{L}$ (NG group) and $33 \mathrm{mmol} / \mathrm{L}$ (HG group) D-glucose for 48 hours. F: Apoptosis rate of HRPE and ARPE-19 cells cultured in $0.3 \mathrm{mmol} / \mathrm{L} \mathrm{H}_{2} \mathrm{O}_{2}$ for 4 hours. G: Relative expression of miR-93 in HRPE and ARPE-19 cells cultured in $0.3 \mathrm{mmol} / \mathrm{L} \mathrm{H}_{2} \mathrm{O}_{2}$ for 4 hours. Data are expressed as means $\pm \mathrm{SD} . n=3(\mathbf{A}-\mathbf{G})$. ${ }^{*} P<0.05$, ${ }^{* * P} P 0.01$, and $* * * P<0.001 ;{ }^{\dagger} P<0.05,{ }^{\dagger \dagger} P<0.01$, and ${ }^{\dagger \dagger} P<0.001$ versus $5 \mathrm{mmol} / \mathrm{L}$. FITC, fluorescein isothiocyanate; HG, high glucose; NG, normal glucose.

transferred onto $0.45-\mu \mathrm{m}$ polyvinylidene difluoride membranes. After the polyvinylidene difluoride membranes had been incubated with primary antibody at $4{ }^{\circ} \mathrm{C}$ overnight, they were then incubated with secondary antibodies. Protein bands were obtained using a FluorChem E System (BioRad, Singapore). An antibody against GAPDH was used for equivalent loading of total proteins. The following antibodies were used: anti-cleaved caspase-3 antibody (ab2302; Abcam, Cambridge, UK), anti-Nrf2 (NFE2L2) antibody (ab137550; Abcam), anti-Bax (BCL2 associated X, apoptosis regulator) antibody (ab32503; Abcam), anti-Bcl-2 (BCL2 apoptosis regulator) antibody (ab32124; Abcam); anti-GAPDH antibody (ab8245; Abcam); and sheep anti-rabbit IgG (horse radish peroxidase) (ab6795; Abcam).

\section{Cell Viability Assay}

ARPE-19 cells were seeded in 96-well culture plates (Corning, Corning, NY); $20 \mu \mathrm{L}$ of MTT (Beyotime Biotech, Shanghai, China) was added to each well, and the plates were then incubated at $37^{\circ} \mathrm{C}$ for another 4 hours. After removing the supernatant, $100 \mu \mathrm{L}$ of dimethyl sulfoxide was added to each well. The optical density at $560 \mathrm{~nm}$ was obtained on a microplate reader (Promega, Madison, WI).

\section{Cell Transfection}

Human miR-93 mimics (5'-CAAAGUGCUGUUCGUGCAGGUAG- $3^{\prime}$ ), normal control (NC) mimics (5'-UGAGACUGGAUGACCAGAGUGG-3'), miR-93 inhibitor (5'-CUACCUGCACGAACAGCACUUUG- $\left.3^{\prime}\right)$, and NC inhibitor (5'-UUCUCCGAACGUGUCACGUTT-3') were obtained from RiBoBio (Guangzhou, China). PcDNA3.1 vector for MEG3 (p-MEG3), siRNA for MEG3 (si-MEG3), pcDNA3.1 vector for Nrf2 (p-Nrf2), pcDNA3.1 vector for $M E G 3 / \mathrm{Nrf2}$ normal control (p-NC), and siRNA for MEG3/ Nrf2 normal control (si-NC) were obtained from RiBoBio. miRNA mimics or a miRNA inhibitor was transfected into the ARPE-19 cells in serum-free Opti-MEM by using Lipofectamine 2000 (Invitrogen, Carlsbad, CA). 

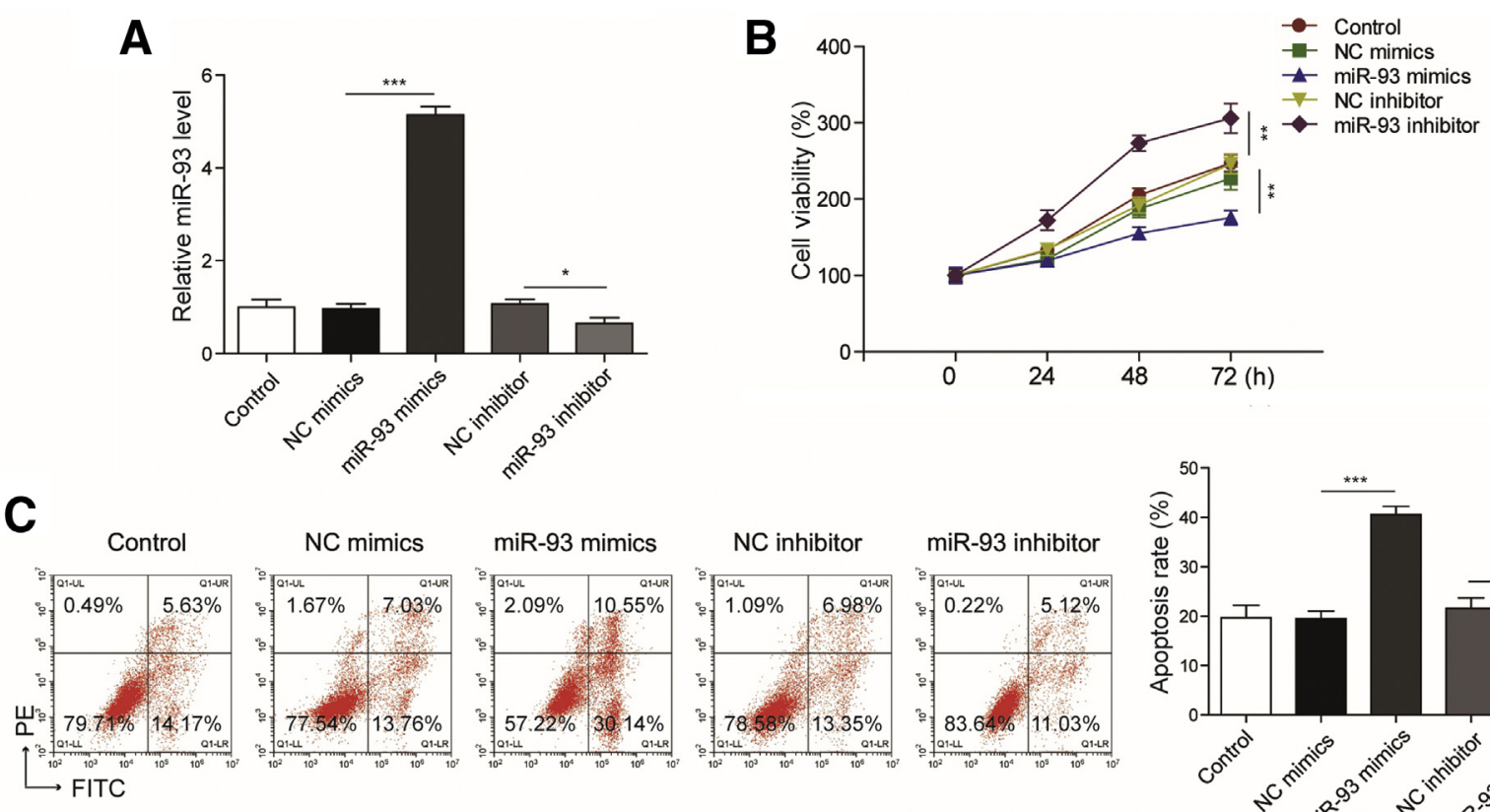

D

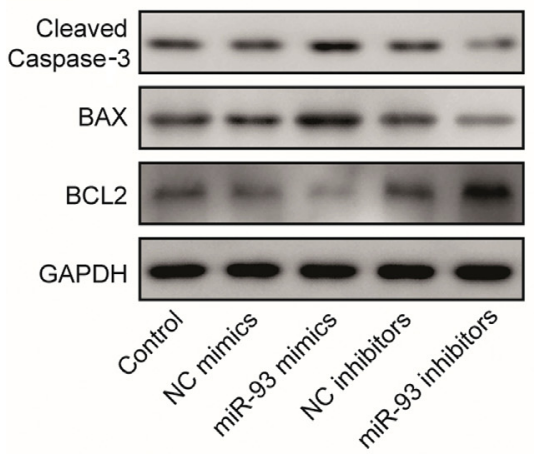

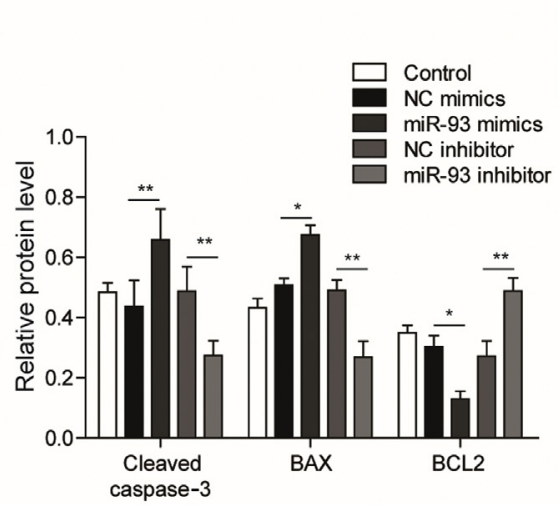

$\mathbf{E}$

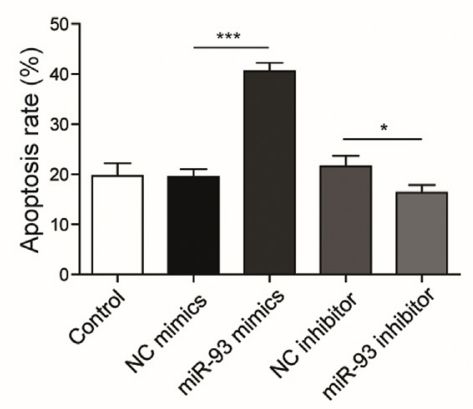

Figure 2 miR-93 (MIR93) promotes high glucose (HG)-induced apoptosis in ARPE-19 cells. A: miR-93 expression in ARPE-19 cells transfected with miR-93 mimics or inhibitor. Quantitative real-time PCR reveals that miR-93 mimics and miR-93 inhibitor work well. B: The cell viability of ARPE-19 cells transfected with miR-93 mimics or miR-93 inhibitor for 0, 24, 48, and 72 hours. C: Apoptosis rate of ARPE-19 cells transfected with miR-93 inhibitor or miR-93 mimics in HG conditions. D: Cell apoptosis related protein [cleaved-caspase 3, BCL2 associated X, apoptosis regulator (BAX), and BCL2 apoptosis regulator (BCL2)] is detected by Western blot. E: Expression of inflammatory factors IL- 6 and tumor necrosis factor (TNF)- $\alpha$ in ARPE-19 cells transfected with miR-93 mimics or inhibitor. Data are expressed as means \pm SD. $n=3$ (A-E). ${ }^{*} P<0.05,{ }^{*} P<0.01$, and ${ }^{* *} P<0.001$. FITC, fluorescein isothiocyanate; GAPDH, glyceraldehyde-3-phosphate dehydrogenase; HG, high glucose; NC, normal control.

\section{Reporter Vector Construction and Luciferase Assay}

To explore the relationship between miR-93-5p and MEG3, miR-93-5p and Nrf2, the reporter vector was constructed by amplifying the $3^{\prime}$-UTR sequence of the MEG3 or Nrf2 gene including miR-93-5p binding sites and inserting into the pMIR-REPORT luciferase vector. To remove the complementarity, the two miR-93-5p complementary sequences UCGUGAAA or GUGAAA in the $3^{\prime}$-UTR were mutated singly or simultaneously. The mutants were called pMIRMEG3/mut and pMIR-Nrf2/mut, respectively. ARPE-19 cells were transfected with the appropriate reporter plasmid and miRNA or expression plasmids in serum-free Opti-MEM by Lipofectamine 2000 (Invitrogen). The luciferase activity was detected on a Dual-Luciferase Reporter Assay System (Promega).

\section{Flow Cytometry Apoptosis Assay}

To explore the interactions among miR-93, IncRNA MEG3, and Nrf2, the apoptosis rate in each group was measured by flow cytometry. The cells were incubated with PIconjugated anti-Annexin V antibodies (Hanbio, Shanghai, China) for 20 minutes under darkness. ${ }^{25}$ The cell apoptosis rate was then measured by flow cytometry (Becton Dickinson, Franklin Lakes, NJ).

\section{ELISA Assay}

Levels of IL- 6 and tumor necrosis factor- $\alpha$ (TNF- $\alpha$ ) were detected by the corresponding enzyme-linked immunosorbent assay (ELISA) kit (Sigma-Aldrich, Darmstadt, Germany) according to the instructions. 

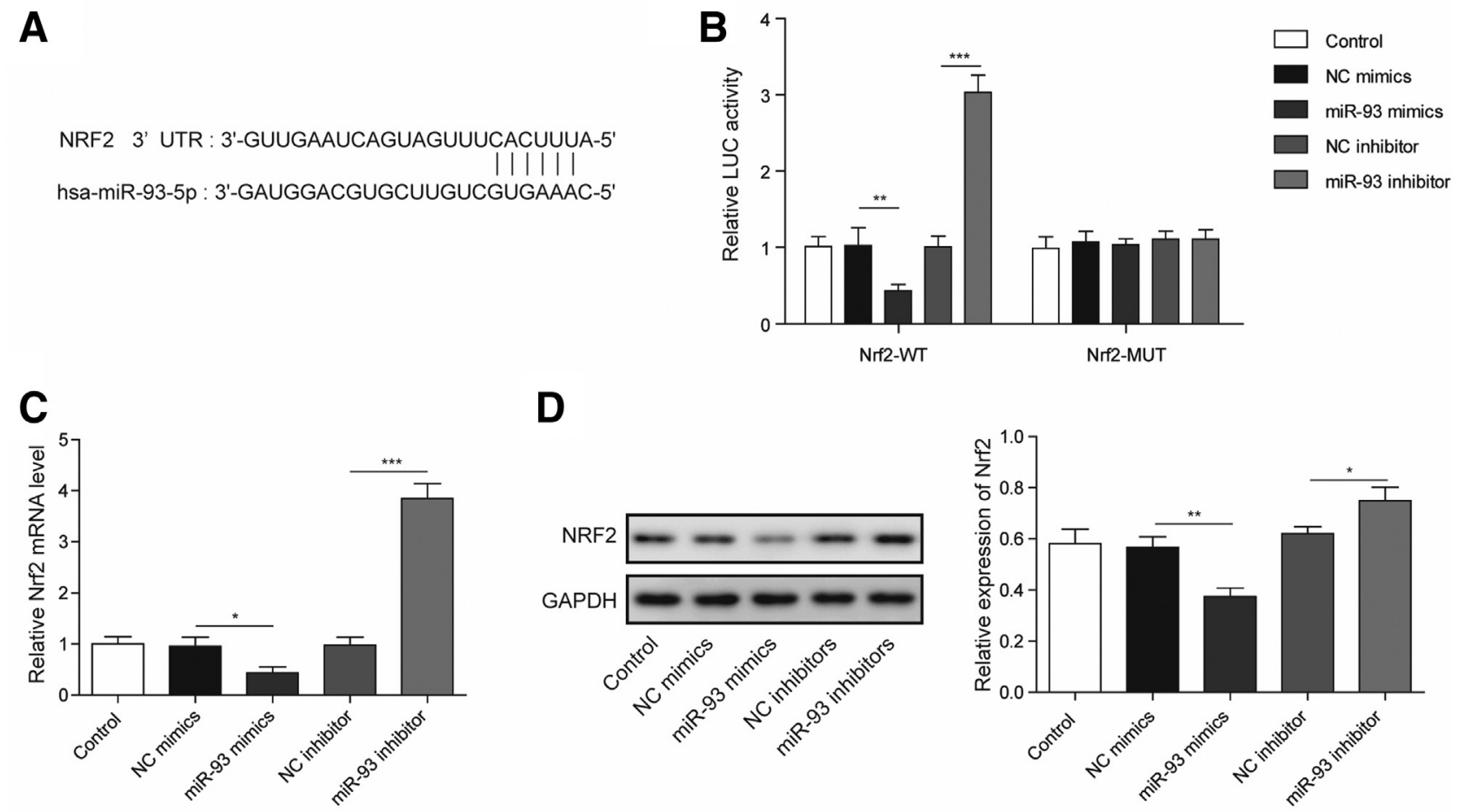

Figure 3 Nrf2 (NFE2L2) is a target gene of miR-93 (MIR93) in ARPE-19 cells. A: Sequence of putative binding site (vertical lines marked) between miR-93 and $3^{\prime}$ untranslated region (UTR)-Nrf2. B: Dual luciferase reporter assay shows that Nrf2 is a target gene of miR-93. C and D: Quantitative real-time PCR (C) and Western blot (D) result show that overexpression of miR-93 markedly inhibits the expression of Nrf2, whereas miR-93 inhibitors significantly promote the expression level of Nrf2. Data are expressed as means \pm SD. $n=3(\mathbf{A}-\mathbf{D}) .{ }^{*} P<0.05,{ }^{*} P<0.01$, and ${ }^{* *} P<0.001$. GAPDH, glyceraldehyde-3-phosphate dehydrogenase; LUC, luciferase; MUT, mutant; NC, normal control; WT, wild type.

\section{Statistical Analysis}

Data are expressed as means $\pm \mathrm{SD}$ and analyzed using SPSS version 14.0 software (SPSS 14.0 for Windows; SPSS Inc., Chicago, IL). Unpaired $t$-test was used for the comparisons between two groups, and the differences among more than two groups were tested by one-way analysis of variance. $P<0.05$ indicates statistical significance.

\section{Results}

HG Damages RPE Cells and Up-Regulates miR-93 Expression

A previous study has shown that the miR-93 is up-regulated during DR. ${ }^{26}$ Therefore, qPCR was used to detect the expression of miR-93 in DR patients and normal subjects. Up-regulated expression of miR-93 was detected in DR patients $(P<0.001)$ (Figure 1A). HRPE and ARPE-19 cells were cultured in $5,15,25,35$, and $45 \mathrm{mmol} / \mathrm{L}$ D-glucose for 48 hours. MTT assay indicated that 25,35 , and $45 \mathrm{mmol} / \mathrm{L} \mathrm{D}_{\mathrm{D}}$ glucose significantly inhibited the activities of HRPE and ARPE-19 cells $(P<0.05)$ (Figure 1B). HRPE and ARPE-19 were cultured in $5 \mathrm{mmol} / \mathrm{L}$ [normal glucose (NG) group] and $33 \mathrm{mmol} / \mathrm{L}$ (HG group) D-glucose for 48 hours. Flow cytometry showed that the apoptosis rate of HRPE and ARPE-19 cells was significantly up-regulated under the HG condition $(P<0.001)$ (Figure $1 \mathrm{C})$. The expression of IL-6 and TNF- $\alpha$ in the NG and HG groups was detected by ELISA. Inflammatory factors were significantly up-regulated in the HG group $(P<0.01)$ (Figure 1D). The expression of miR-93 was detected by qPCR, and results indicate that the expression of miR-93 increased in HRPE and ARPE-19 cells cultured with HG $(P<0.05)$ (Figure 1E). Moreover, HRPE and ARPE-19 were cultured in $0.3 \mathrm{mmol} / \mathrm{L} \mathrm{H}_{2} \mathrm{O}_{2}$ for 4 hours, and apoptosis was detected by flow cytometry. The results showed that $\mathrm{H}_{2} \mathrm{O}_{2}$ induced apoptosis significantly $(P<0.01)$ (Figure 1F). In terms of the expression of miR-93, the results from qPCR suggest that there is no significant difference between the control group and the $\mathrm{H}_{2} \mathrm{O}_{2}$ group (not significant) (Figure 1G). Together, the data illustrate that HG stimulated expression of miR-93 in DR.

\section{miR-93 Is Up-Regulated under HG Condition}

To explore the effects of miR-93 on HG-induced apoptosis in RPE cells, miR-93 mimics or miR-93 inhibitors were transfected into ARPE-19 cells. Results from qPCR revealed that miR-93 mimics and miR-93 inhibitor worked well $(P<0.05)$ (Figure 2A). The cell viability of the above cells was further measured by MTT. It was found that overexpression of miR-93 markedly suppressed the viability of HG-treated cells, whereas knockdown of miR-93 enhanced it $(P<0.01)$ (Figure 2B). Apoptosis was detected by flow 

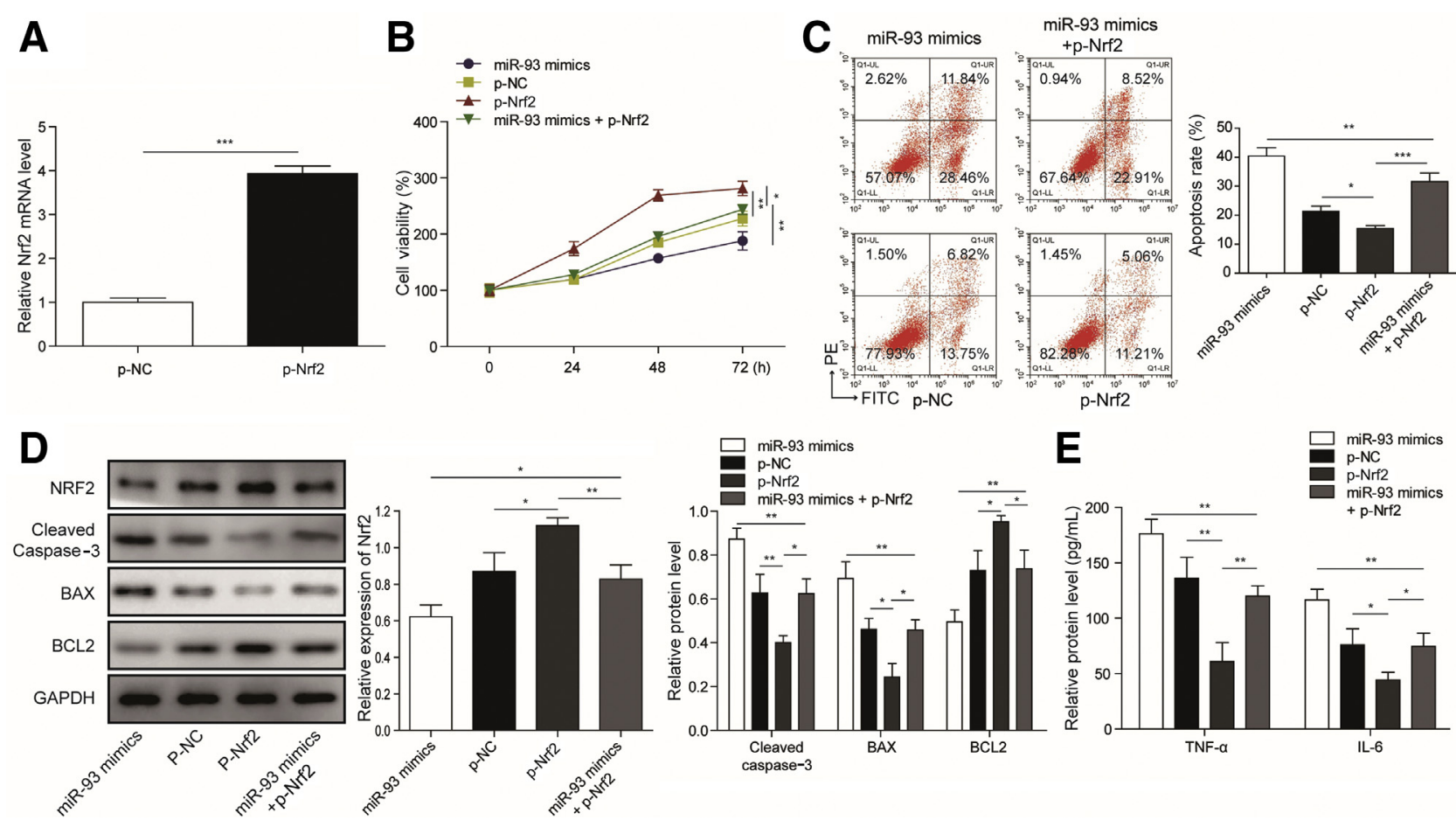

Figure 4 Nrf2 (NFE2L2) overexpression alleviates the high glucose-induced apoptotic effect of ARPE-19 cells mediated by miR-93 (MIR93). A: Transfection efficiency of overexpression plasmid detected by quantitative real-time PCR. B: The cell viability of ARPE-19 cells transfected with p-Nrf2 and miR-93 mimics for $0,24,48$, and 72 hours. C: Apoptosis rate of ARPE-19 cells transfected with p-Nrf2 and miR-93 mimics. D: Western blot results indicate that overexpression of Nrf2 significantly inhibits the cleaved-caspase 3 and BCL2 associated $X$, apoptosis regulator (BAX), and promotes the expression of BCL2 apoptosis regulator (BCL2), and overexpression of Nrf2 reverses the effect of miR-93 on the promotion of cleaved-caspase 3 and Bax, and the inhibitory effect on Bcl-2. E: The expression of IL- 6 and tumor necrosis factor (TNF)- $\alpha$ is detected by enzyme-linked immunosorbent assay (ELISA). The results show that Nrf2 can inhibit the secretion of inflammatory cytokines induced by high glucose, and overexpression of Nrf2 reverses the proinflammatory effect of overexpressing miR-93. The experiments were performed under high glucose $(\mathrm{HG})$ conditions. Data are expressed as means \pm SD. $n=3(\mathbf{A}-\mathbf{E}) .{ }^{*} P<0.05,{ }^{* *} P<0.01$, and ${ }^{* * *} P<0.001$. FITC, fluorescein isothiocyanate; GAPDH, glyceraldehyde-3-phosphate dehydrogenase.

cytometry. Overexpression of miR-93 significantly promoted apoptosis induced by HG $(P<0.001)$, whereas inhibiting the expression of miR-93 alleviated HG-induced apoptosis $(P<0.05)$ (Figure 2C). Concurrently, proapoptotic protein caspase-3 and Bax were significantly increased in the miR-93 mimics group $(P<0.01 ; P<0.05)$, and down-regulated in the miR-93 inhibitors group $(P<0.01)$; antiapoptotic protein Bcl-2 was remarkably down-regulated in the miR-93 mimics group $(P<0.05)$ and up-regulated in the miR-93 inhibitors group $(P<0.01)$ (Figure $2 \mathrm{D})$. The expression of IL-6 and TNF- $\alpha$ was detected by ELISA. The results showed that overexpression of mir-93 promoted the secretion of inflammatory factors, whereas knockdown of mir-93 inhibited the expression of inflammatory factors $(P<0.05)$ (Figure $2 \mathrm{E}$ ). The above results suggest that miR-93 aggravates HG-induced apoptosis and inflammation.

\section{Nrf2 Is a Target Gene of miR-93 in ARPE-19 Cells}

To elucidate the latent mechanism of miR-93 overexpression aggravating HG-induced RPE apoptosis, miRNA target prediction was performed using StarBase v2.0. ${ }^{27}$ Consistent with the literature, this study found the binding site of miR-93 and Nrf2 (Figure 3A). Further, a high level of miR-93 reduced the fluorescence intensity of wildtype Nrf2 in ARPE-19 cells, and silencing of miR-93 led to opposite results $(P<0.01)$, but little effect was seen on the fluorescence intensity of Mut-Nrf2 (not significant) (Figure 3B). Moreover, qPCR and Western blot results showed that up-regulation of miR-93 inhibited the expression of Nrf2, whereas miR-93 inhibitors significantly increased the expression of Nrf2 $(P<0.05)$ (Figure 3, C and $\mathrm{D})$. These results indicated that miR-93 directly targets and negatively regulated Nrf2.

Nrf2 Overexpression Alleviates the HG-Induced
Apoptotic Effect of ARPE-19 Cells Mediated by miR-93

The overexpression vector of Nrf2 (p-Nrf2) and its empty vector (p-NC) was transfected into the cells. Increased expression of $\mathrm{Nrf} 2$ was observed in p-Nrf2 group $(P<0.001)$ (Figure 4A). MTT assay showed that upregulation of Nrf2 increased cell viability and reversed the inhibitory effect of miR-93 on cell viability $(P<0.05)$ (Figure 4B). Further, overexpression of Nrf2 significantly inhibited HG-induced apoptosis $(P<0.05)$ and reversed the proapoptotic effect of miR-93 $(P<0.001)$ (Figure $4 \mathrm{C})$. The expression of Nrf2 was detected by Western blot. The 

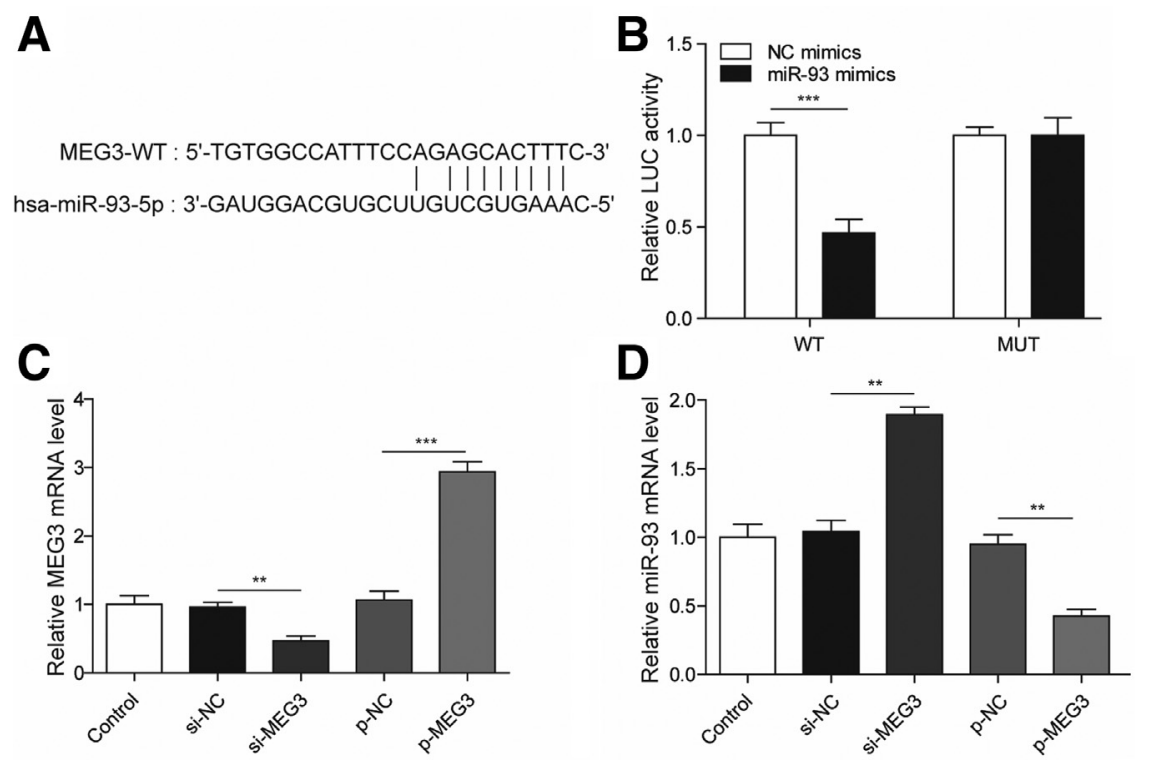

Figure $5 \quad M E G 3$ directly targets miR-93 (MIR93) in ARPE-19 cells. A: Sequence of putative binding site (vertical lines marked) between miR-93 and IncRNA MEG3. B: Results of the DualLuciferase Reporter Assay show that MEG3 directly targets miR-93 in ARPE-19 cells. C: The transfection efficiency of si-MEG3 and $p-M E G 3$ detected by quantitative real-time PCR. si-MEG3 significantly inhibits the expression of MEG3, whereas $\mathrm{p}-M E G 3$ promotes the expression of MEG3. D: Relative expression of miR-93 after the transfection of si-MEG3 and $\mathrm{p}-M E G 3$ in ARPE-19 cells. si-MEG3 significantly promotes whereas p-MEG3 significantly inhibits the expression of miR-93. Data are expressed as means \pm SD. $n=3$ (A-D). ${ }^{* *} P<0.01,{ }^{* * *} P<0.001$. LUC, luciferase; MUT, mutant; NC, normal control; WT, wild type.

results from this analysis showed that overexpression of miR-93 inhibited the expression of Nrf2 and reversed the effect of Nrf2 overexpression on the protein level of Nrf2 $(P<0.05)$ (Figure 4D). Overexpression of Nrf2 inhibited the expression of cleaved caspase 3 and Bax, and promoted the expression of $\mathrm{Bcl}-2$; and overexpression of $\mathrm{Nrf} 2$ reversed the effect of miR-93 on the promotion of cleaved caspase 3 and Bax, and the inhibitory effect on Bcl-2 $(P<0.05)$ (Figure 4D). The expression of inflammatory factors IL- 6 and TNF- $\alpha$ was detected by ELISA. The results showed that Nrf2 can inhibit the secretion of inflammatory factors induced by $\mathrm{HG}$, and overexpression of $\mathrm{Nrf} 2$ reversed the proinflammatory effect of miR-93 overexpression $(P<0.05)$ (Figure 4E). The above results indicate that Nrf2 overexpression could attenuate miR-93-mediated apoptosis induced by $\mathrm{HG}$ in retinal pigment epithelial cells.

\section{MEG3 Directly Targets miR-93 in ARPE-19 Cells}

Complementary binding sites between MEG3 and miR-93 were found according to bioinformatics analysis (Figure 5A). To verify their correlation, wild-type $M E G 3$ and Mut-MEG3 luciferase reporter plasmids were designed. Fluorescence intensity was significantly lower in the ARPE19 cells co-transfected with wild-type MEG3 and miR-93 mimics than cells co-transfected with Mut-MEG3 and miR-93 mimics $(P<0.001)$ (Figure 5B). qPCR results illustrated that si-MEG3 significantly inhibited the expression of MEG3 $(P<0.01)$, whereas p-MEG3 promoted the expression of $M E G 3(P<0.001)$ (Figure $5 \mathrm{C})$. Further, after transfection of si-MEG3, the expression of miR-93 significantly increased $(P<0.01)$, whereas overexpression of MEG3 significantly inhibited the expression of miR-93
$(P<0.01)$ (Figure 5D). The above results indicate that in ARPE-19, MEG3 targets and negatively regulates miR-93.

MEG3 Is Down-Regulated under HG Condition, Overexpression of MEG3 Alleviates HG-Induced Apoptosis through the miR-93/Nrf2 Axis

The expression of $M E G 3$ was measured in DR patients and healthy controls, and a lower level of $M E G 3$ was discovered in DR patients $(P<0.01)$ (Figure 6A). HRPE and ARPE-19 cells were cultured with NG and HG, respectively. qPCR results showed that $M E G 3$ was significantly down-regulated in the HG group $(P<0.01)$ (Figure 6B). The HG treatment groups were: control, si-NC, si-MEG3, p-NC, p-MEG3, and p-MEG3+miR-93 mimics. The results revealed that the expression of $N r f 2$ mRNA was down-regulated when MEG3 was knocked down $(P<0.01)$ and up-regulated when $M E G 3$ was overexpressed $(P<0.001)$, but the regulation of $N r f 2$ by MEG3 was reversed by overexpression of miR-93 $(P<0.001)$ (Figure 6C). MTT assay showed that knockdown of MEG3 increased the inhibitory effect of $\mathrm{HG}$ on cell viability $(P<0.01)$, whereas overexpression of $M E G 3$ reduced the induction of $\mathrm{HG}$ on cell viability $(P<0.01)$; overexpression of miR-93 reversed the promoting effect of $M E G 3$ on cell viability $(P<0.05)$ (Figure $6 \mathrm{D})$. The expression of Nrf2 in ARPE-19 cells after 48 hours of si-MEG3, p-MEG3, and p-MEG3+miR-93 mimics transfection was detected by Western blot. The results revealed that the expression of Nrf2 was down-regulated when MEG3 was knocked down$(P<0.01)$ and up-regulated when $M E G 3$ was overexpressed $(P<0.01)$, but the regulation of Nrf2 by $M E G 3$ was reversed by overexpression of miR-93 $(P<0.05)$ (Figure $6 \mathrm{E})$. Flow cytometry showed that knockdown of $M E G 3$ aggravated HG-induced apoptosis $(P<0.01)$; overexpression of MEG3 

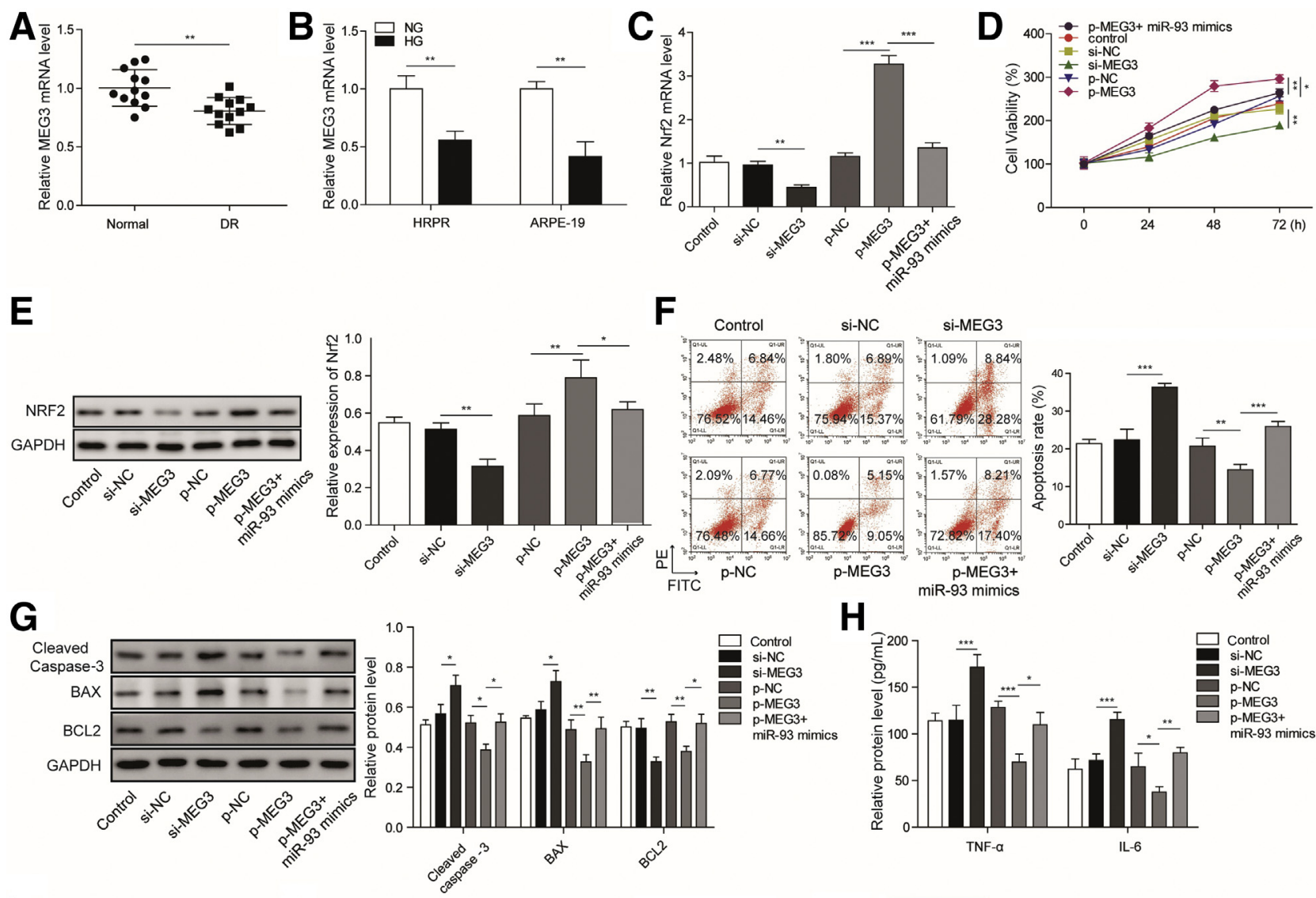

H

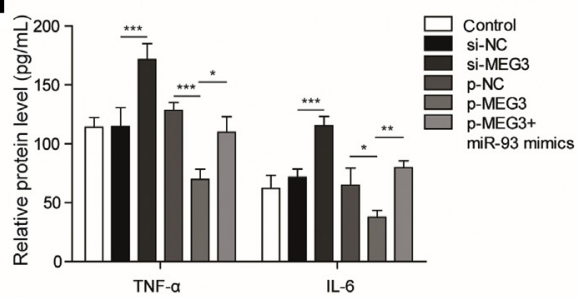

I

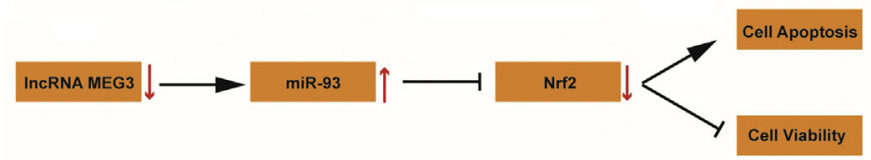

Figure 6 MEG3 is down-regulated under the high glucose (HG) condition; overexpression of MEG3 alleviates high-glucose (HG)-induced apoptosis through the miR-93(MIR93)/Nrf2(NFE2L2) axis. A: Relative expression of MEG3 in diabetic retinopathy (DR) patients and healthy controls. B: Relative expression of MEG3 in HRPE and ARPE-19 cells treated with normal and high glucose for 48 hours (NG and HG). C: The expression of Nrf2 in ARPE-19 cells after 48 hours of siMEG3, p-MEG3, and p-MEG3+miR-93 mimics transfection detected by quantitative real-time PCR. D: The cell viability of ARPE-19 cells after $0,24,48$, and 72 hours of si-MEG3, p-MEG3, and p-MEG3+miR-93 mimics transfection measured by MTT. E: The expression of Nrf2 in ARPE-19 cells after 48 hours of si-MEG3, pMEG3, and p-MEG3+miR-93 mimics transfection detected by Western bolt. F: Apoptosis is detected by flow cytometry in ARPE-19 cells after 48 hours of siMEG3, p-MEG3, and p-MEG3 + miR-93 mimics transfection. G: Western blot results exhibit that knockdown of MEG3 increases the expression of cleaved caspase 3 and $\mathrm{BCL2}$ associated $\mathrm{X}$, apoptosis regulator (BAX), and decreases the expression of BCL2 apoptosis regulator (BCL2); overexpression of MEG3 down-regulates the expression of cleaved caspase 3 and BAX and up-regulates the expression of BCL2, whereas overexpression of miR-93 reverses this trend. H: IL-6 and tumor necrosis factor (TNF)- $\alpha$ are detected by enzyme-linked immunosorbent assay (ELISA) in ARPE-19 cells after 48 hours of si-MEG3, p-MEG3, and p-MEG3+ miR-93 mimics transfection. I: IncRNA-MEG3 alleviates high glucose-induced apoptosis of retinal epithelial cells via regulating the miR-93/Nrf2 axis. Data are expressed as means \pm SD. $n=3(\mathbf{A}-\mathrm{I}) .{ }^{*} P<0.05,{ }^{*} P<0.01$, and ${ }^{* *} P<0.001$. FITC, fluorescein isothiocyanate; GAPDH, glyceraldehyde-3-phosphate dehydrogenase.

attenuated HG-induced apoptosis $(P<0.001)$, and overexpression of miR-93 reversed the antiapoptotic effect of MEG3 $(P<0.01)$ (Figure 6F). Western blot results revealed that knockdown of $M E G 3$ increased the expression of cleaved caspase 3 and Bax, and decreased the expression of Bcl-2. Overexpression of $M E G 3$ depressed the expression of cleaved caspase 3 and Bax, and up-regulated the expression of Bcl-2, whereas overexpression of miR-93 reversed this trend $(P<0.05)$ (Figure 6G). The results of ELISA demonstrated that knockdown of MEG3 stimulated the expression of IL-6 and TNF- $\alpha$, whereas overexpression of MEG3 showed the opposite results. However, overexpression of miR-93 reversed the anti-inflammatory effect of $M E G 3(P<0.05)$ (Figure $6 \mathrm{H})$. The above results indicate that $M E G 3$ was down-regulated under the HG condition; overexpression of $M E G 3$ alleviated HG-induced apoptosis and inflammation through the miR-93/ Nrf2 axis (Figure 6I).

\section{Discussion}

The main problem with DR is the occurrence of metabolic, vascular, and neurological complications. ${ }^{1}$ RPE cell apoptosis is an early event in DR. ${ }^{28}$ In the current study, 
up-regulated expression of miR-93 was noticed in DR patients, as well as in HG-exposed HRPE and ARPE-19 cells, indicating that miR-93 plays an potential role in occurrence and development of DR. Therefore, the function and regulation mechanism of miR-93 were further studied.

MiR-93 often functions as a promoter of tumor progression in several human carcinomas such as glioblastoma and breast cancer. ${ }^{29,30}$ Moreover, Hirota et al ${ }^{24}$ pointed out that miR-93 was highly expressed in patients with proliferative DR. A previous study ${ }^{31}$ demonstrated that plasma miR-93 participated in the type $2 \mathrm{DR}$ progression. Consistent with previous studies, ${ }^{24,31}$ up-regulated miR-93 was observed in DR patients, and HG-exposed HRPE and ARPE-19 cells, indicating that miR-93 may be a potential key factor involved in DR progression.

Although apoptosis has been proved to be one of the main characteristics of DR progression in RPE cells, there is no direct evidence that the up-regulation of miR-93 has any effect on RPE. In the previous literature, the regulatory role of miR-93 in apoptosis is controversial. miR-93 was upregulated in oxygen and glucose deprivation/reoxygenation model, and miR-93 inhibition ameliorated oxygen and glucose deprivation/reoxygenation-induced hypoxia/reoxygenation injury apoptosis in cells by targeting Nrf2. ${ }^{32}$ However, in cardiomyocytes, miR-93-3p functioned as a protective factor against inflammation and apoptosis. ${ }^{33}$ Therefore, the expression of miR-93 is inconsistent under different disease states, sometimes manifested as proapoptosis and sometimes as antiapoptosis. The current study confirmed that miR-93 inhibited the viability and increased apoptosis and inflammation in HG-exposed ARPE-19 cells.

This study further investigated how miR-93 regulates apoptosis in RPE cells. It is well documented that Nrf2 plays a critical protective role against oxidative stress ${ }^{34}$ and inflammation. ${ }^{35}$ In addition, Niture and Jaiswal ${ }^{36}$ have found that $\mathrm{Nrf} 2$ protein could up-regulate antiapoptotic protein $\mathrm{Bcl}-2$ and prevent cellular apoptosis. Another study has indicated that $\mathrm{Nrf} 2$ is an important protective factor regulating the progression of DR, and it suggested that up-grading of the Nrf2 pathway may act as a potential therapeutic method for DR. ${ }^{37}$ Evidence concerning a potential protective role for Nrf2 in the retina is increasing. ${ }^{38}$ Consistent with the previous literature, the present study revealed that overexpression of Nrf2 can reverse the proapoptotic effect of miR-93.

As a tumor suppressor gene, functionality of $M E G 3$ has been widely studied in different types of human cancer. ${ }^{39,40}$ A previous study pointed out that the expression of MEG3 was down-regulated in HG-treatment ARPE-19 cells, $M E G 3$ overexpression reduced the increased expression levels of VEGF and TGF- $\beta 1$ induced by HG treatment. ${ }^{41}$ Therefore, it was concluded that lncRNA MEG3 overexpression may inhibit the development of DR by inhibiting TGF- $\beta 1$ and VEGF expression. ${ }^{41}$ Consistent with previous reports, down-regulated $M E G 3$ expression was detected in DR patients, and HG-exposed HRPE and ARPE-19 cells. Therefore, the authors speculated that MEG3 may play a potential regulatory part during DR. A study on diabetic mice showed significantly down-regulated expression of $M E G 3$ and aggravated retinal vascular dysfunction. ${ }^{20}$ However, it is unclear whether MEG3 can regulate apoptosis in RPE cells. Wang et $\mathrm{al}^{42}$ uncovered that lncRNA MEG3 could inhibit apoptosis in ethanol-induced AML-12 cells and hepatic steatosis. Another study suggested that inhibition of IncRNA MEG3 was conducive to protect rat brain microvascular endothelial cells against oxygen and glucose deprivation/reoxygenation-induced apoptosis. ${ }^{43}$ Consistent with the above studies, this current study reveals that in HG-cultured ARPE-19 cells, MEG3 enhanced cell viability and inhibited apoptosis and inflammation, and it is further demonstrated that $M E G 3$ functions through the miR-93/Nrf2 axis.

To sum up, the present study demonstrates that lncRNA MEG3 and Nrf2 were decreased and miR-93 was increased under DR and HG conditions. Nrf2 is a target gene of miR-293, Nrf2 overexpression alleviates the HG-induced apoptotic effect of ARPE-19 cells mediated by miR-93. MEG3 directly targets miR-93, overexpression of MEG3 alleviates HG-induced apoptosis and inflammation through the miR-93/Nrf2 axis. A mouse model of DR will be established to investigate the role of these genes/proteins in the development of DR in future studies by the authors.

\section{References}

1. Bourne RR, Stevens GA, White RA, Smith JL, Flaxman SR, Price H, Jonas JB, Keeffe J, Leasher J, Naidoo K, Pesudovs K, Resnikoff S, Taylor HR; Vision Loss Expert Group: Causes of vision loss worldwide, 1990-2010: a systematic analysis. Lancet Glob Health 2013, 1:e339-e349

2. Cheung N, Mitchell P, Wong TY: Diabetic retinopathy. Lancet 2010, 376:124-136

3. Prasad PS, Schwartz SD, Hubschman J-P: Age-related macular degeneration: current and novel therapies. Maturitas 2010, 66:46-50

4. Abramoff MD, Garvin MK, Sonka M: Retinal imaging and image analysis. IEEE Rev Biomed Eng 2010, 3:169-208

5. Franco LM, Zulliger R, Wolf-Schnurrbusch UEK, Katagiri Y, Kaplan HJ, Wolf S, Enzmann V: Decreased visual function after patchy loss of retinal pigment epithelium induced by low-dose sodium iodate. Invest Ophthalmol Vis Sci 2009, 50:4004-4010

6. Vinores SA, Derevjanik NL, Ozaki H, Okamoto N, Campochiaro PA: Cellular mechanisms of blood-retinal barrier dysfunction in macular edema. Doc Ophthalmol 1999, 97:217-228

7. Wang J, Xu X, Elliott MH, Zhu M, Le Y-Z: Muller cell-derived VEGF is essential for diabetes-induced retinal inflammation and vascular leakage. Diabetes 2010, 59:2297-2305

8. Xu HZ, Le Y-Z: Significance of outer blood-retina barrier breakdown in diabetes and ischemia. Invest Ophthalmol Vis Sci 2011, 52: 2160-2164

9. Weinberger D, Fink-Cohen S, Gaton DD, Priel E, Yassur Y: Nonretinovascular leakage in diabetic maculopathy. $\mathrm{Br} \mathrm{J}$ Ophthalmol 1995, 79:728-731

10. Aizu Y, Oyanagi K, Hu J, Nakagawa H: Degeneration of retinal neuronal processes and pigment epithelium in the early stage of the streptozotocin-diabetic rats. Neuropathology 2002, 22:161-170

11. Vinores SA, Van Niel E, Swerdloff JL, Campochiaro PA: Electron microscopic immunocytochemical demonstration of blood-retinal barrier breakdown in human diabetics and its association with 
aldose reductase in retinal vascular endothelium and retinal pigment epithelium. Histochem J 1993, 25:648-663

12. Vinores SA, Van Niel E, Swerdloff JL, Campochiaro PA: Electron microscopic immunocytochemical evidence for the mechanism of blood-retinal barrier breakdown in galactosemic rats and its association with aldose reductase expression and inhibition. Exp Eye Res 1993, 57:723-735

13. Kirber WM, Nichols CW, Grimes PA, Winegrad AI, Laties AM: A permeability defect of the retinal pigment epithelium. Occurrence in early streptozocin diabetes. Arch Ophthalmol 1980, 98:725-728

14. Lin X, Zhou X, Liu D, Yun L, Zhang L, Chen X, Chai Q, Li L: MicroRNA-29 regulates high-glucose-induced apoptosis in human retinal pigment epithelial cells through PTEN. In Vitro Cell Dev Biol Anim 2016, 52:419-426

15. Holtkamp GM, Kijlstra A, Peek R, de Vos AF: Retinal pigment epithelium-immune system interactions: cytokine production and cytokine-induced changes. Prog Retin Eye Res 2001, 20:29-48

16. Kim DI, Park MJ, Lim SK, Choi JH, Kim JC, Han HJ, Kundu TK, Park J, Yoon KC, Park SW, Park JS, Heo YR, Park SH: High-glucoseinduced CARM1 expression regulates apoptosis of human retinal pigment epithelial cells via histone 3 arginine 17 dimethylation: role in diabetic retinopathy. Arch Biochem Biophys 2014, 560:36-43

17. Simo R, Villarroel M, Corraliza L, Hernandez C, Garcia-Ramirez M: The retinal pigment epithelium: something more than a constituent of the blood-retinal barrier-implications for the pathogenesis of diabetic retinopathy. J Biomed Biotechnol 2010, 2010:190724

18. Perkel JM: Visiting "noncodarnia". Biotechniques 2013, 54. 301, 303-304

19. Jae N, Dimmeler S: Long noncoding RNAs in diabetic retinopathy. Circ Res 2015, 116:1104-1106

20. Qiu G-Z, Tian W, Fu H-T, Li C-P, Liu B: Long noncoding RNAMEG3 is involved in diabetes mellitus-related microvascular dysfunction. Biochem Biophys Res Commun 2016, 471:135-141

21. Mastropasqua R, Toto L, Cipollone F, Santovito D, Carpineto P, Mastropasqua L: Role of microRNAs in the modulation of diabetic retinopathy. Prog Retin Eye Res 2014, 43:92-107

22. Ye P, Liu J, He F, Xu W, Yao K: Hypoxia-induced deregulation of miR-126 and its regulative effect on VEGF and MMP-9 expression. Int J Med Sci 2014, 11:17-23

23. Mortuza R, Feng B, Chakrabarti S: miR-195 regulates SIRT1-mediated changes in diabetic retinopathy. Diabetologia 2014, 57:1037-1046

24. Hirota K, Keino H, Inoue M, Ishida H, Hirakata A: Comparisons of microRNA expression profiles in vitreous humor between eyes with macular hole and eyes with proliferative diabetic retinopathy. Graefes Arch Clin Exp Ophthalmol 2015, 253:335-342

25. Zheng S-Z, Sun P, Wang J-P, Liu Y, Gong W, Liu J: MiR-34a overexpression enhances the inhibitory effect of doxorubicin on HepG2 cells. World J Gastroenterol 2019, 25:2752-2762

26. Kumar M, Nath S, Prasad HK, Sharma GD, Li Y: MicroRNAs: a new ray of hope for diabetes mellitus. Protein Cell 2012, 3:726-738

27. Yang J-H, Li J-H, Shao P, Zhou H, Chen Y-Q, Qu L-H: starBase: a database for exploring microRNA-mRNA interaction maps from Argonaute CLIP-Seq and Degradome-Seq data. Nucleic Acids Res 2011, 39:D202-D209

28. Song MK, Roufogalis BD, Huang TH: Reversal of the caspasedependent apoptotic cytotoxicity pathway by taurine from Lycium barbarum (Goji berry) in human retinal pigment epithelial cells: potential benefit in diabetic retinopathy. Evid Based Complement Alternat Med 2012, 2012:323784

29. Fang L, Deng Z, Shatseva T, Yang J, Peng C, Du WW, Yee AJ, Ang LC, He C, Shan SW, Yang BB: MicroRNA miR-93 promotes tumor growth and angiogenesis by targeting integrin-beta8. Oncogene 2011, 30:806-821

30. Fang L, Du WW, Yang W, Rutnam ZJ, Peng C, Li H, O'Malley YQ, Askeland RW, Sugg S, Liu M, Mehta T, Deng Z, Yang BB: MiR-93 enhances angiogenesis and metastasis by targeting LATS2. Cell Cycle 2012, 11:4352-4365

31. Zou HL, Wang Y, Gang Q, Zhang Y, Sun Y: Plasma level of miR-93 is associated with higher risk to develop type 2 diabetic retinopathy. Graefes Arch Clin Exp Ophthalmol 2017, 255:1159-1166

32. Yan L-J, Fan X-W, Yang H-T, Wu J-T, Wang S-L, Qiu C-G: MiR-93 inhibition ameliorates OGD/R induced cardiomyocyte apoptosis by targeting Nrf2. Eur Rev Med Pharmacol Sci 2017, 21:5456-5461

33. Tang B, Xuan L, Tang M, Wang H, Zhou J, Liu J, Wu S, Li M, Wang X, Zhang H: miR-93-3p alleviates lipopolysaccharide-induced inflammation and apoptosis in $\mathrm{H} 9 \mathrm{c} 2$ cardiomyocytes by inhibiting toll-like receptor 4. Pathol Res Pract 2018, 214:1686-1693

34. Ruiz S, Pergola PE, Zager RA, Vaziri ND: Targeting the transcription factor Nrf2 to ameliorate oxidative stress and inflammation in chronic kidney disease. Kidney Int 2013, 83:1029-1041

35. Kim J, Cha Y-N, Surh Y-J: A protective role of nuclear factorerythroid 2-related factor-2 (Nrf2) in inflammatory disorders. Mutat Res 2010, 690:12-23

36. Niture SK, Jaiswal AK: Nrf2 protein up-regulates antiapoptotic protein Bcl-2 and prevents cellular apoptosis. J Biol Chem 2012, 287: 9873-9886

37. Xu Z, Wei Y, Gong J, Cho H, Park JK, Sung E-R, Huang H, Wu L, Eberhart C, Handa JT, Du Y, Kern TS, Thimmulappa R, Barber AJ, Biswal S, Duh EJ: NRF2 plays a protective role in diabetic retinopathy in mice. Diabetologia 2014, 57:204-213

38. Wei Y, Gong J, Yoshida T, Eberhart CG, Xu Z, Kombairaju P, Sporn MB, Handa JT, Duh EJ: Nrf2 has a protective role against neuronal and capillary degeneration in retinal ischemia-reperfusion injury. Free Radic Biol Med 2011, 51:216-224

39. Qin R, Chen Z, Ding Y, Hao J, Hu J, Guo F: Long non-coding RNA MEG3 inhibits the proliferation of cervical carcinoma cells through the induction of cell cycle arrest and apoptosis. Neoplasma 2013, 60: 486-492

40. Zhou Y, Zhang X, Klibanski A: MEG3 noncoding RNA: a tumor suppressor. J Mol Endocrinol 2012, 48:R45-R53

41. Zhang D, Qin H, Leng Y, Li X, Zhang L, Bai D, Meng Y, Wang J: LncRNA MEG3 overexpression inhibits the development of diabetic retinopathy by regulating TGF-beta1 and VEGF. Exp Ther Med 2018, 16:2337-2342

42. Wang Q, Li M, Shen Z, Bu F, Yu H, Pan X, Yang Y, Meng X, Huang C, Li J: The long non-coding RNA MEG3/miR-let-7c-5p axis regulates ethanol-induced hepatic steatosis and apoptosis by targeting NLRC5. Front Pharmacol 2018, 9:302

43. Zhan R, Xu K, Pan J, Xu Q, Xu S, Shen J: Long noncoding RNA MEG3 mediated angiogenesis after cerebral infarction through regulating p53/NOX4 axis. Biochem Biophys Res Commun 2017, 490:700-706 